des sonst so bequem liegenden Hauptsterns a Arietis, und endlich das Zusammenfallen mit dem Orte des Planeten selbst, liess für die ersten Sterne nur mit Mühe die Zahl von 4 Bestimmungen erreichen. Auch die innere Harmonie der Beobachtungen lässt $z u$ wünschen ubrig. Aber in dieser Beziehung war bei den starken Temperaturumschlägen und den durchaus nur sehr mittelmässigen Bildern auch kaum mehr zu erwarten. Eine Ausnahme machen hiervon nur die Sterne des Normalbogens und einige der letzten schon im October absolvirten. Für die Sterne des Normalbogens

Pulkowa 1889 März I I. ergiebt sich der wahrsch. Fehler einer Beobachtung \pm 0.044 resp. \pm 0.32 , während derselbe aus allen Beobachtungen \pm 0 . 059 resp. \pm 0.52 folgt. Ich muss bemerken, dass ich mich gezwungen sah, einige Declinationsbestimmungen wegen gar zu starker Abweichung, die sehr wahrscheinlich eine Folge der Verstellung des Instruments bei nicht ganz hinreichender Klemmung war, auszuschliessen. Es geschah dieses bei den Sternen, wo die Zahl der Bestimmungen für $\alpha$ und $\delta$ verschieden angegeben sind. Die Grössen sind die der Durchnusterung.

\title{
Observations of Comet 1888 III
}

on the Equatorial of Morrison Observatory, Glasgow, Missouri, U.S.A., by C. W. Pritchett.

\begin{tabular}{|c|c|c|c|c|c|c|c|c|c|c|c|c|c|c|c|}
\hline & Berlin M.I. & $\Delta \alpha$ & \multicolumn{2}{|c|}{$\Delta \delta$} & 'Cp. & \multicolumn{2}{|r|}{$\alpha$ app. } & $g_{1}$ & \multicolumn{3}{|c|}{$\delta$ app. } & $\log p . A$ & \multicolumn{2}{|l|}{ Red. ad l. app. } \\
\hline & 3 & $15^{\mathrm{h}} 5^{2^{\mathrm{m}}} 37^{\mathrm{s}}$ & $\mathrm{J}$ & & & 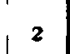 & $10^{h}$ & $\mathrm{~m}$ & . & & & & & 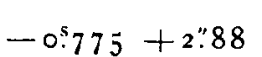 & \\
\hline & 23 & $15 \quad 22 \quad 12$ & - I 45.84 & $\cdots 2$ & .40 & 3 & 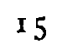 & 5 & & & 39 & & & $+0.135+$ & \\
\hline & 24 & 15 II 48 & +73 & +3 & & 4 & 15 & 9 & & & 55 & & & 6 & \\
\hline & 25 & $14 \quad 58 \quad 24$ & -13 & +1 & 4 & 9 & I & I325: & 8 & & 12 & & & $8-$ & \\
\hline & 27 & 145148 & -324 & +0 & & 7 & 5 & $205^{8}$ & & & 47 & & & $24-$ & \\
\hline & 29 & $1448 \quad 28$ & -343 & $\cdots-3$ & 3 & 9 & I 5 & 273 & & & 25 & & & $5-$ & \\
\hline & 30 & $14 \quad 53 \quad 24$ & +430 & +5 & & 7 & I 5 & 305 & & +14 & 44 & & & $9+$ & \\
\hline 6. & $\mathbf{I}$ & $143^{8} 3^{2}$ & -344 & +3 & & 8 & 15 & 34 I & & & 5 & & 0.6 & 4 & \\
\hline & 2 & $1453 \quad 38$ & to 57 & +12 & & 8 & I 5 & 3728 & & & 25. & $345^{8}$ & & 45 & \\
\hline & 6 & $14 \quad 45 \quad 43$ & $+345.3^{6}$ & +3 & 2.48 & 5 & $\sqrt{5}$ & 4950 & 1 & +10 & 55 & & 0.708 & $7+$ & \\
\hline & 7 & $\begin{array}{lll}14 & 45 & 27 \\
14 & 48 & 46\end{array}$ & $\begin{array}{r}-58.67 \\
+638.09\end{array}$ & $\begin{array}{l}+0 \\
+5\end{array}$ & $\begin{array}{l}54.57 \\
50.05\end{array}$ & 5 & I 5 & $\begin{array}{ll}52 & 49.34 \\
55 & 43.60\end{array}$ & $\begin{array}{l}9.65^{\circ} \\
0.652\end{array}$ & to & 19 & $\begin{array}{l}5^{8.54} \\
50.63\end{array}$ & & $+0.300+8.69$ & \\
\hline
\end{tabular}

Mean Places of the Comparison stars.

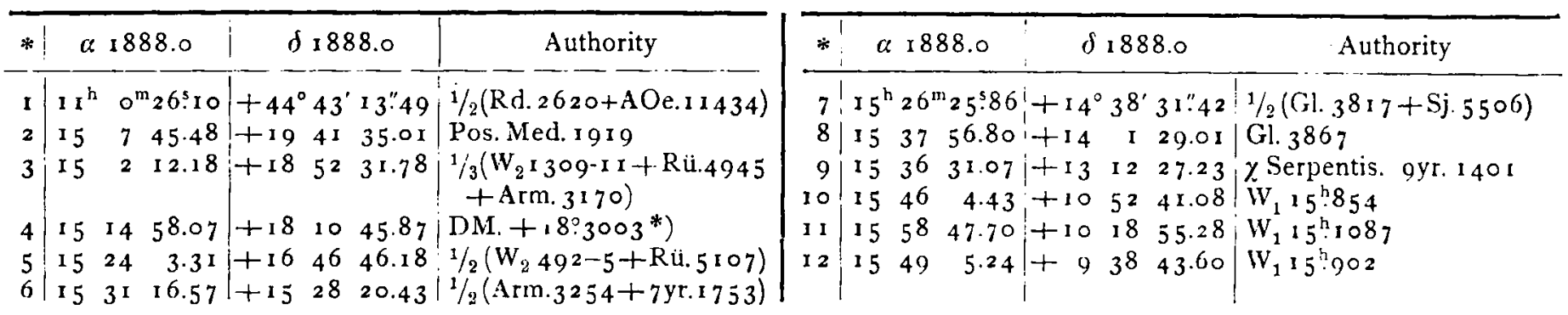

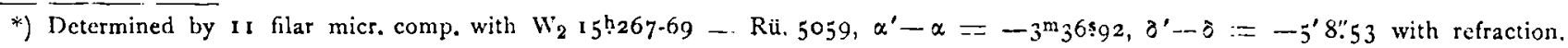

Note. The later observations were somewhat difficult; but it could have been accurately observed a week longer, if the weather and moon had allowed.

\section{Neuer Comet Barnard 1889 Juni 23.}

Am 25. Juni Morgens erhielt die Centralstelle folgendes Telegramm aus Boston:

Faint Comet Barnard June 23.9499 Gr. M.T. RA. app. $=20^{\circ} 1^{\prime} 21^{\prime \prime}$ PD. app. $=51^{\circ} 9^{\prime} 1^{\prime \prime}$.

Daily motion $+1^{\circ} 6^{\prime}$ in RA., $-0^{\circ} 34^{\prime}$ in $\mathrm{PI}$.

Jen Sternwarten der südlichen Halbkugel wurde dies Telegramm nicht nitgetheilt.

Kr.

\section{In halt:}

7u Nr. 2905-06. F. Coniel. Coordonnés héliocentriques de Jupiter, 1. - E.S. Holdin. Auszug aus einem Schreiben betr. den Schweif des. Crmeten 1889 I. 27. - H. Romberg. Beobachtungen von (7) Iris und Vergleichsternen während der Opposition 1888, 29. C. W. Pritchett. Observations of Comet I 888 III. 3I. - Neuer Comet Barnard 1889 Juni 23. 31 